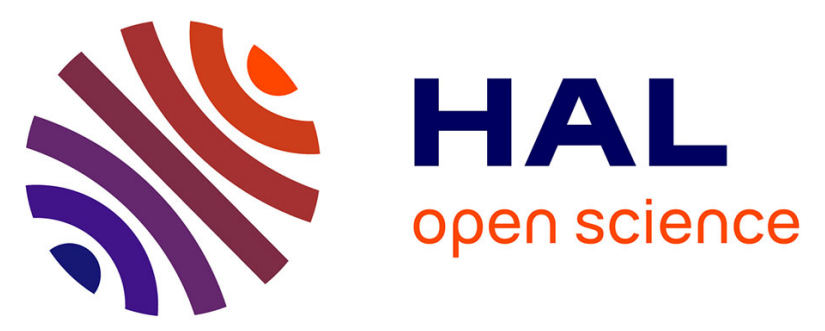

\title{
Optically Transparent Flexible Robust Circularly Polarized Antenna for UHF RFID Tags
}

Abu Sadat Md. Sayem, Duc Le, Roy B. V. B. Simorangkir, Toni Bjorninen, Karu P. Esselle, Raheel M. Hashmi, Maxim Zhadobov

\section{- To cite this version:}

Abu Sadat Md. Sayem, Duc Le, Roy B. V. B. Simorangkir, Toni Bjorninen, Karu P. Esselle, et al.. Optically Transparent Flexible Robust Circularly Polarized Antenna for UHF RFID Tags. IEEE Antennas and Wireless Propagation Letters, 2020, 19 (12), pp.2334-2338. 10.1109/LAWP.2020.3032687 . hal-02996701

\section{HAL Id: hal-02996701 https://hal-univ-rennes1.archives-ouvertes.fr/hal-02996701}

Submitted on 9 Nov 2020

HAL is a multi-disciplinary open access archive for the deposit and dissemination of scientific research documents, whether they are published or not. The documents may come from teaching and research institutions in France or abroad, or from public or private research centers.
L'archive ouverte pluridisciplinaire HAL, est destinée au dépôt et à la diffusion de documents scientifiques de niveau recherche, publiés ou non, émanant des établissements d'enseignement et de recherche français ou étrangers, des laboratoires publics ou privés. 


\title{
Optically Transparent Flexible Robust Circularly Polarized Antenna for UHF RFID Tags
}

\author{
Abu Sadat Md. Sayem, Graduate Student Member, IEEE, Duc Le, Graduate Student Member, IEEE, Roy B. V. B. \\ Simorangkir, Member, IEEE, Toni Björninen, Senior Member, IEEE, Karu P. Esselle, Fellow, IEEE, Raheel M. \\ Hashmi, Member, IEEE and Maxim Zhadobov, Senior Member, IEEE
}

\begin{abstract}
Optically transparent flexible tag antennas are highly desired for some applications, where unobtrusiveness and capability of mounting on curved surfaces are required. On the other hand, circular polarization minimizes signal degradation due to polarization mismatch between the reader and tag antennas, thus ensuring good communication quality and accuracy. In this paper, we present an optically transparent and flexible radio frequency identification (RFID) passive tag antenna operating in the ultra-high frequency (UHF) band and exhibits circular polarization. It is thin, lightweight and completely encapsulated inside transparent polymer which protects it against dust, water, heat and mechanical stress. The tag antenna is made by utilizing highly flexible and transparent conductive-meshpolymer composite through a simple and low-cost manufacturing process. The antenna design is based on a planar square-ring. It achieves a measured maximum read range of about $8.3 \mathrm{~m}$ and approximately $47 \mathrm{MHz}(885-932 \mathrm{MHz})$ 3-dB axial-ratio bandwidth.
\end{abstract}

Index Terms-Circular polarization, flexible, radio frequency identification (RFID), polymer composite, tag antenna, textile, ultra-high frequency (UHF).

\section{INTRODUCTION}

In recent years, radio frequency identification (RFID) technology operating in the ultra-high frequency (UHF) band has attracted considerable attention due to the excellent features of long reading range, high data rate and small size. UHF RFID systems have enormous applications in wireless detection and tracking operations, including object identification, electronic toll collection, health-care, distributed sensor networks, access control, supply chain management and detection of fraudulent activities in retail shops, factories and warehouses [1], [2].

In passive UHF RFID systems, tag antennas among other factors determine the read range and data accuracy. Circularly polarized (CP) tag antennas are preferred over linearly polarized (LP) tag antennas because of their less sensitivity to multipath effects caused by reflections from ambient objects

This work was supported in part by the Australian Commonwealth Government funded International Research Training Program Scholarship in Macquarie University, Australia. (Corresponding author: Abu Sadat Md. Sayem.)

A. S. M. Sayem, R. B. V. B. Simorangkir and R. M. Hashmi are with the School of Engineering, Macquarie University, Sydney, Australia. (E-mail: abu-sadat-md-.sayem@students.mq.edu.au).

T. Björninen and D. Le are with Faculty of Medicine and Health Technology, Tampere University, Tampere 33720, Finland. (E-mail: vietduc. le@tuni.fi).

K. P. Esselle is with the School of Electrical \& Data Engineering, University of Technology Sydney, Australia. (E-mail: Karu.Esselle@uts.edu.au).

M. Zhadobov is with the University of Rennes 1, CNRS, Institut d'Electronique et de Télécommunications of Rennes (IETR)-UMR 6164, F35000 Rennes, France. (E-mail: maxim.zhadobov@univ-rennes1.fr).
[3]. Hence, CP tags maintain stable communication links with the readers even when the orientation of the tags changes. This is particularly important when tags are deployed on moving objects. Moreover, most of the commercial RFID technologies use $\mathrm{CP}$ reader antennas. When the polarization orientation of the CP tag antenna is perfectly matched with the $\mathrm{CP}$ reader antenna, then the polarization efficiency will be equivalent to 1 , which leads to nearly $41 \%$ (optimum) improvement in the attainable read range over an LP tag antenna [4]. In addition, with the exponentially rising demands of RFID technology, new requirements on tag antennas' characteristics have also been imposed. For instance, tag antennas integrated on vehicle windshields [3] need to be transparent for enhancing aesthetic. Similarly, tag antennas used for providing protection against counterfeiting in retail industry should be visually imperceptible. Unnoticeable tag antennas are also required in involuntary treatment and dementia care as well as in any other applications which value unobtrusiveness for users' convenience and aesthetic. Optical transparency is considered as a good form of achieving unobtrusiveness in antennas' visual appearance [5]. It can also be noted that for the deployment on human body or any platforms with curved surfaces, tag antennas should be flexible and concurrently robust against physical deformation [6]. So, it is ascertained that conformal transparent CP tag antennas have numerous applications. Methods to develop CP tag antennas can be found in the literature [3], [4], [7]-[10] but the development of a flexible, robust and transparent $\mathrm{CP}$ tag antenna has not been addressed.

The realization of flexible and optically transparent tag antennas is, however, very challenging due to the unavailability of suitable materials and complex fabrication processes. In the traditional transparent thin films [11]-[13] and meshed conductors [14], [15], there is a trade-off between the optical and electrical properties [16], hence, high optical transparency and good RF performance cannot be achieved simultaneously. Moreover, complex and costly fabrication techniques are required to fabricate antennas with these materials. It is also challenging to maintain robust integration of these transparent conductors with flexible substrates in harsh physical deformations [17].

Recently, conductive-mesh-polymer composite [18] has been presented as an excellent candidate for the realization of flexible transparent antennas, demonstrating some interesting features of high flexibility, robustness and ease of fabrication. This composite material has therefore been selected to realize the proposed tag antenna. 
In this paper, a new flexible UHF RFID CP tag antenna is introduced. It exhibits high optical transparency, flexibility, good reading distance and $\mathrm{CP}$ bandwidth, and comprises a simple and planar structure. For designing transparent antennas, the geometry occupying large open area is the appropriate choice. For this reason, ring-shaped geometry has been utilized for the demonstrated tag antenna. Moreover, with planar ring antennas, good axial ratio (AR) bandwidth can be achieved [3], [4] and its planar structure makes it easy to manufacture. The performance of the proposed antenna was examined through fabricating a prototype and measuring its read range and $\mathrm{CP}$ performance in both unbent and bent states. The prototype shows robust performance when exposed to physical deformations, demonstrating its suitability for ubiquitous applications.

\section{ANTENNA GEOMETRY AND DESIGN CONSIDERATIONS}

The topology of the demonstrated CP tag antenna is shown in Fig. 1. The antenna accomplishes a narrow square-ring, loaded with meander strip, feeding strip, and matching strip. In simulation, the antenna was excited with a $50-\Omega$ excitation port and post-processing was applied for further investigations of the tag antenna performance (e.g., input reflection coefficient and read range) for actual IC impedance. To note that, the port is an external structure which does not affect the antenna characteristics, such as input impedance, current distribution, directivity and radiation pattern. The selected width of the ring is $2 \mathrm{~mm}$ which is optimized to maintain larger opening for better transparency while giving good reading range. The meander strip is introduced in the antenna to reduce its size. This meander strip also disturbs the induced electric field along the $x$ - and $y$-axes in the quadrature phase [3] and, thus, excites CP radiation. The associated feeding strip along with an open gap maintains CP radiation. By optimizing the length, $l_{\mathrm{f}}$ and width, $w_{\mathrm{f}}$ of the feeding strip, position of the IC, $p$, position of the gap, $l_{\mathrm{x}}$ and width of the gap, $g, \mathrm{CP}$ radiation is excited in the desired operating band. The directions of the simulated current distribution of the antenna at the time phases $0^{\circ}, 90^{\circ}, 180^{\circ}$ and $270^{\circ}$ are shown in Fig. 2, which indicates that the antenna is circularly polarized. Fig. 3 depicts the simulated radiation patterns of the antenna in $\mathrm{xz}-$ and $\mathrm{yz}-$ planes, demonstrating that the antenna radiates bidirectionally with a left-hand circular polarization (LHCP) towards the front side of the antenna (i.e., positive z-axis direction) and a righthand circular polarization (RHCP) towards the back side of the antenna. The simulated 3-dB AR beamwidths of the antenna at $900 \mathrm{MHz}$ are approximately $75^{\circ}$ and $50^{\circ}$ in xz- and yz-planes, respectively, which demonstrates a wide angular span of the CP performance.

The conductive parts of the antenna are fabricated with flexible conductive sheet, VeilShield from Less EMF Inc. VeilShield is a very thin $(57 \mu \mathrm{m})$ conductive sheet having low sheet resistance $(0.1 \Omega / s q)$. The top and bottom PDMS layers serve as the protective covers of the antenna. PDMS solution was prepared by using Dow Corning Sylgard 184 silicone elastomer kit, maintaining 10:1 base to curing agent mixing ratio. By using Agilent $85070 \mathrm{E}$ Dielectric Kit, the measured dielectric constant of the PDMS was found 2.75

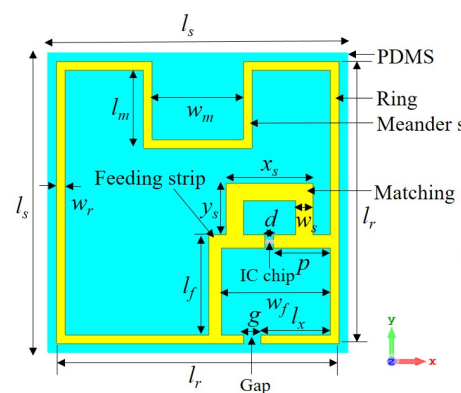

(a)

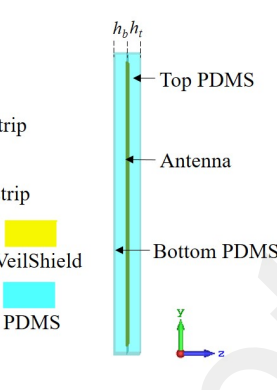

(b)
Fig. 1. Antenna configuration: (a) Top view, (b) Side view. $\left(l_{r}=65, l_{s}=\right.$ 69, $w_{r}=2, l_{m}=18, w_{m}=21, l_{x}=16, \mathrm{~g}=4, l_{f}=23, w_{f}=25, d=2, p$ $=13, x_{s}=20, y_{s}=12, w_{s}=4, h_{b}=0.5, h_{t}=0.5$ ). (All dimensions are in $\mathrm{mm})$.

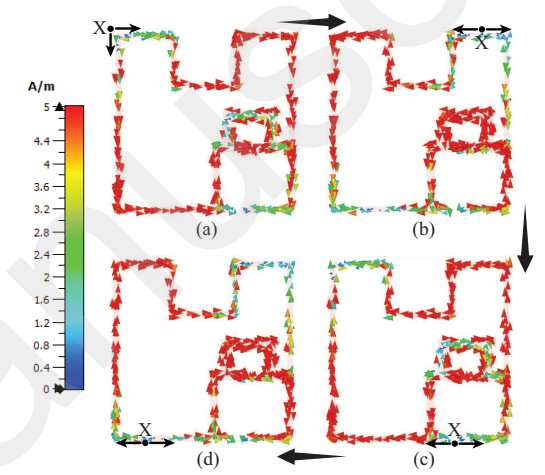

Fig. 2. Predicted current distribution of the tag antenna at $900 \mathrm{MHz}$ at the time phases: (a) $0^{\circ}$, (b) $90^{\circ}$, (c) $180^{\circ}$ and (d) $270^{\circ}$.

which was nearly invariable in the conducted measurement from 0.5 to $10.6 \mathrm{GHz}$, whereas the loss tangent varied from 0.008 to 0.07 in this frequency range. The thickness of the tag antenna with PDMS encapsulation is $1 \mathrm{~mm}$. VeilShield itself is not robust but after forming the composite with PDMS, it turns into a highly flexible and robust material [18]. The NXP UCODE G2iL series RFID microchip is used as the tag IC in this design, which has a wake-up power of $-18 \mathrm{dBm}$ $(15 \mu \mathrm{W})$. The IC is mounted by the manufacturer on a copper fixture patterned on plastic film. The $3 \mathrm{~mm} \times 3 \mathrm{~mm}$ pad of the fixture is attached to the antenna with silver conductive epoxy. The tag IC along with the copper fixture is modelled in the simulations as the parallel connection of a $2.85 \mathrm{k} \Omega$ resistor and a $0.91 \mathrm{pF}$ capacitor [6]. At $900 \mathrm{MHz}$, the impedance of the equivalent circuit of the IC is approximately 13.2-193j

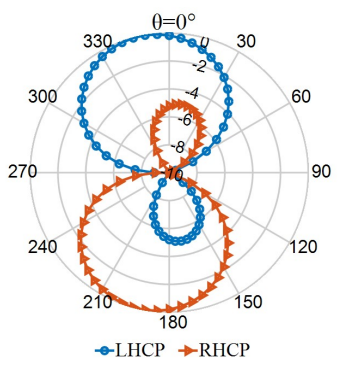

(a)

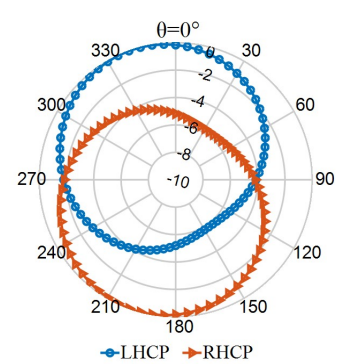

(b)
Fig. 3. Simulated radiation patterns of the antenna at $900 \mathrm{MHz}$ : (a) xz-plane and (b) yz-plane. 
$\Omega$. For optimum power transfer between the antenna and the tag chip, tag antenna's input impedance should be conjugate matched with the tag IC's input impedance. However, input impedance of a square-ring antenna is high [19]. For proper impedance matching, the matching strip is introduced to form a closed loop around the feeding points to reduce the input resistance [3] to match it with the IC. Input impedance of the tag antenna can be tuned and matched to tag IC by optimizing the parameters $x_{s}, y_{s}$ and $w_{s}$. However, from the investigation, it is found that there is a trade-off among conjugate impedance matching, read range and polarization state. In this work, the antenna design parameters are optimized for maximizing the tag read range while having a good $\mathrm{CP}$ in the target operating band instead of focusing on achieving an optimal impedance matching.

In free-space, the attainable read range of the tag in the directions $\phi$ and $\theta$ of a spherical coordinate system is computed in the simulations by using the formula given in [20]:

$$
d_{\text {tag }}(\phi, \theta)=\frac{\lambda}{4 \pi} \sqrt{\frac{\chi_{p o l}(\phi, \theta) \tau e_{r} D(\phi, \theta) E I R P}{P_{i c 0}}}
$$

where $\lambda$ is the free-space wavelength of the reader's carrier signal, $\chi_{p o l}$ is the mutual polarization power efficiency between the tag and reader antennas, $e_{r}$ is the radiation efficiency of the tag antenna, $D$ is the directivity of the tag antenna, $E I R P$ is the equivalent isotropic radiated power of the reader, $P_{i c 0}$ is the wake-up power of the tag IC and $\tau$ is the power transmission coefficient which accounts for the impedance mismatch between the antenna $\left(Z_{\text {ant }}\right)$ and IC $\left(Z_{i c}\right)$. Power transmission coefficient, $\tau$ is determined by the following equation:

$$
\tau=\frac{4 R e\left(Z_{a n t}\right) \operatorname{Re}\left(Z_{i c}\right)}{\left|Z_{a n t}+Z_{i c}\right|^{2}}
$$

In simulations, $d_{\text {tag }}$ was calculated under the European RFID emission regulation: EIRP $=3.28 \mathrm{~W}$, assuming perfect polarization matching between the reader and tag antennas: $\chi_{\text {pol }}=1$.

The photograph of the fabricated prototype shown in Fig. 4(a) demonstrates its high transparency by the clear visibility of the printed text through it. The area occupied by the ring and feeding structure (i.e., nearly $18 \%$ of the total area of the antenna) has about $70 \%$ optical transparency based on an investigation under a Carry 5000 UV-vis-NIR spectrophotometer from Agilent, and the rest of the antenna, composed of PDMS, has approximately 94\% transparency [21]. In Fig. 4(b), the antenna under extreme bending is shown, demonstrating its excellent flexibility. The prototype was fabricated by following the same layer-by-layer manufacturing procedure using ringshaped molds as described in our previous work [18].

\section{MEASUREMENTS AND RESULTS}

\section{A. Measurement procedure}

The performance of the fabricated tag antenna was experimentally characterized in terms of its reading range and axial

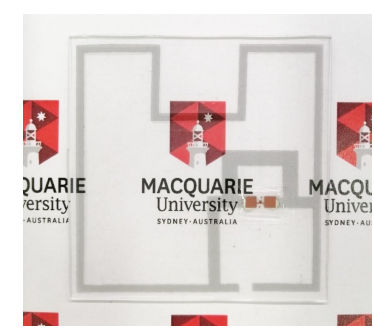

(a)

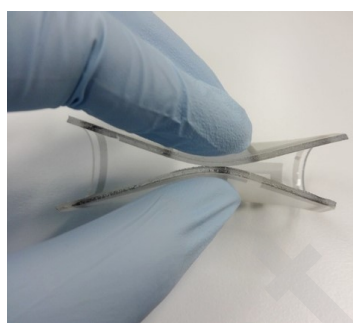

(b)
Fig. 4. Photographs of the fabricated prototype: (a) unbent state, (b) bent state.

ratio. The measurements were conducted upon a fully assembled tag in an anechoic chamber with Voyantic Tagformance system based on a reader-to-tag communication threshold. It is worth to mention the advantages of this approach. Firstly, there is no need to measure the tag antenna and the IC separately. Secondly, it takes into account the IC mounting parasitics correctly and automatically [22]. The performance of the proposed tag antenna were measured in unbent state and different bending states. The performance under bent states were observed to explore its suitability in conformal environment. The performance under bending was assessed with the antenna conformed over a hollow cylindrical structure having the radius of approximately $30 \mathrm{~mm}$ and $40 \mathrm{~mm}$ in both $x$-axis (horizontal) and $y$-axis (vertical) directions. Moreover, the antenna was measured when bent along diagonal axis with a bending radius of approximately $40 \mathrm{~mm}$. The bending test set-up is shown in the inset of Fig. 5.

For measuring the antenna's axial ratio (AR), we used an LP antenna at the reader module of the system and recorded the threshold power $\left(P_{t h}\right)$ at various rotating angles of the tag in $x y$-plane (see the inset of Fig. 5). Here, $P_{t h}$ is defined as the lowest transmission power at which the tag remains responsive, i.e., being able to send a valid 16-bit random number as a response to a query command in ISO 180006C communication standard. Both the tag and reader antennas were positioned at the same height and the rotating angle resolution of the tag was $10^{\circ}$. At each frequency, the AR was calculated from the maximum and minimum values of $P_{t h}$ over the whole rotation angles with the following relationship [23]:

$$
A_{t a g}=\sqrt{\frac{P_{t h, \max }}{P_{t h, \min }}}
$$

After measuring the axial ratio of the antenna, we measured the read range of the antenna by using a $\mathrm{CP}$ reader antenna. The attainable read range $\left(d_{t a g}\right)$ of the tag is estimated from its measured threshold power in accordance with the following equation [24]:

$$
d_{t a g}=\frac{\lambda}{4 \pi} \sqrt{\frac{E I R P}{\Lambda} \frac{P_{t h *}}{P_{t h}}}
$$

where $\Lambda$ and $P_{t h *}$ are the sensitivity and measured threshold power of the reference tag, respectively. The sensitivity $\Lambda$ comes from the manufacturer and $P_{t h *}$ is the threshold power of the system reference tag measured by us (this is the part 


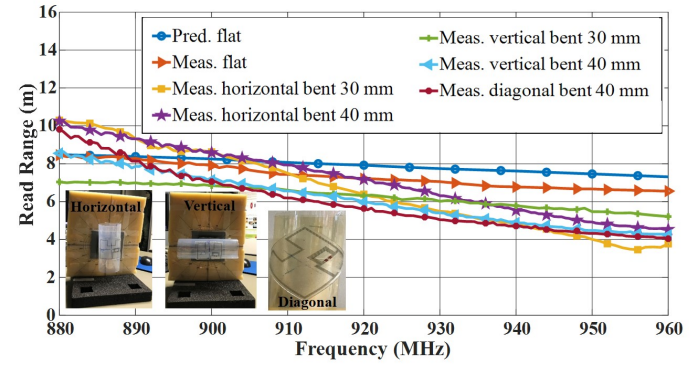

Fig. 5. Read range of the CP tag antenna in flat state and various bending configurations. The inset shows the bending test set-up.

of the calibration procedure we undertook each time before starting the measurement of the actual tag under test). In Eq. (4), $d_{t a g}$ describes the maximum theoretical distance between the tag and reader in free space environment. Since the reference tag is linearly polarized, we divided the $P_{t h *}$ with a factor of two to compensate the linear-to-circular polarization mismatch loss.

\section{B. Results}

The predicted attainable read range in flat state derived from simulation and the measured results in both flat and bent states are shown in Fig. 5 in the frequency range 880-960 $\mathrm{MHz}$. The illustrated results in Fig. 5 depict very good agreement between the predicted and measured flat state results. The small discrepancy in results comes from the inaccuracy in manual fabrication process as well as the attachment of the tag IC to the antenna by using silver epoxy. In unbent state, the measured read range at the target frequency of $900 \mathrm{MHz}$ is approximately $7.3 \mathrm{~m}$. The read range performance of this transparent flexible tag antenna is comparable to rigid opaque tag antennas [3], [7]-[10]. The attainable read range of the tag antenna under different bending states are shown in Fig. 5, which exhibits the variations in the read range across the observation frequency. The variations in read range under bending states happen possibly due to the changes in the antenna physical structure under deformation, affecting the overall antenna characteristics (i.e., input impedance, directivity, radiation pattern, and axial ratio) and their frequency trends. The bending test results depict that under the bending at small radii, the antenna read range performance remains satisfactory.

The predicted and measured axial ratio (AR) in the flat state are depicted in Fig. 6 and a good agreement can be observed between them. A small variation comes from the fabrication error. As AR is highly sensitive to the antenna geometry, specially feeding strip, any little discrepancy in feeding structure affects the AR performance severely. The measured results of Fig. 6 show that the proposed tag antenna has excellent $\mathrm{CP}$ performance in the operating UHF band. The measured 3-dB AR bandwidth (BW) of the antenna in flat state is $47 \mathrm{MHz}(885-932 \mathrm{MHz})$. Fig. 6 also illustrates the AR performance under different bent states. In $x$-axis (horizontal) bending, the 3-dB AR bandwidth are $35 \mathrm{MHz}(882-917 \mathrm{MHz})$ and $46 \mathrm{MHz}(882-928 \mathrm{MHz})$ in $30 \mathrm{~mm}$ and $40 \mathrm{~mm}$ bending radius, respectively. In $y$-axis (vertical) bending, the 3 -dB AR

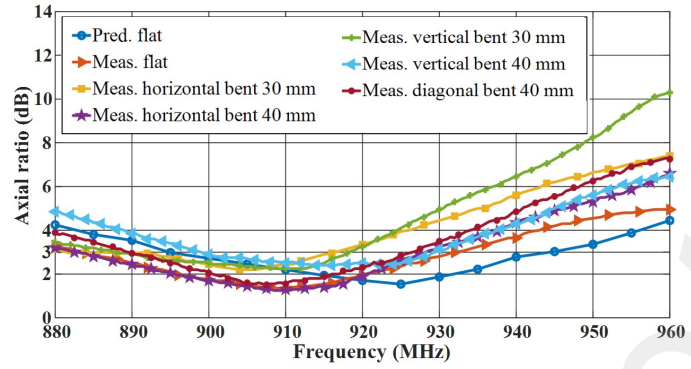

Fig. 6. Axial ratio (AR) of the $\mathrm{CP}$ tag antenna in flat state and various bending configurations.

TABLE I

COMPARISON OF THE DEMONSTRATED CP TAG ANTENNA WITH OTHER STATE-OF-THE-ART UHF CP TAG ANTENNAS

\begin{tabular}{l|l|l|l|l}
\hline Ref. & Size $\left(\mathrm{mm}^{2}\right)$ & $\begin{array}{l}\text { Max. Read } \\
\text { Range }(\mathrm{m})\end{array}$ & $\begin{array}{l}\text { 3-dB } \\
\text { AR BW } \\
(\mathrm{MHz})\end{array}$ & $\begin{array}{l}\text { Gain at } \\
915 \mathrm{MHz} \\
(\mathrm{dBic})\end{array}$ \\
\hline$[3]$ & $54 \times 54$ & 8.3 & 36 & 1.8 \\
\hline$[4]$ & $90 \times 90$ & 16.3 & 50 & 2.83 \\
\hline$[7]$ & $36.5 \times 36.5$ & 7.6 & 11.4 & NA \\
\hline$[8]$ & $70 \times 70$ & 2 & 6 & -13 \\
\hline$[9]$ & $100 \times 100$ & 4.1 & 20 & -8.5 \\
\hline$[10]$ & $127.9 \times 189.6$ & 8 & 15 & 6 \\
\hline Proposed & $69 \times 69$ & 8.3 & 47 & -1.05 \\
\hline
\end{tabular}

bandwidth are $31 \mathrm{MHz}(887-918 \mathrm{MHz})$ and $32 \mathrm{MHz}(897-$ $929 \mathrm{MHz}$ ) in $30 \mathrm{~mm}$ and $40 \mathrm{~mm}$ bending radius, respectively. In diagonal bending, the 3-dB AR bandwidth is $37 \mathrm{MHz}(889-$ $926 \mathrm{MHz}$ ). From the bending test results, it can be concluded that the new tag antenna is well suited for flexible operations.

Finally, the performance of the new antenna is compared with a few other state-of-the-art UHF CP tag antennas in Table I. As can be seen, the read range and $\mathrm{CP}$ performance of the new tag antenna are comparable to the previously reported $\mathrm{CP}$ tag antennas which are all rigid and opaque. Therefore, the proposed tag antenna can be a potential candidate for conformal transparent CP RFID operations.

\section{CONCLUSION}

A highly flexible, robust and transparent passive UHF CP tag antenna has been demonstrated in this paper. This PDMS encapsulated tag antenna is realized from transparent and flexible conductive-mesh-polymer composite. The new antenna can be used in many applications where the RFID tags are frequently exposed to harsh mechanical stress. The CP nature of the tag antenna makes it suitable for many applications, especially where the tag is mounted on moving objects and the orientation between the tag and reader is likely to mismatch. Due to conformal structure, the developed antenna can be mounted on both flat and curved surfaces. The prototype has demonstrated a very good read range, comparable to some rigid $\mathrm{CP}$ tag antennas, and good $\mathrm{CP}$ bandwidth in both flat and bent states. In flat state, the maximum measured attainable read range and 3-dB AR bandwidth are $8.3 \mathrm{~m}$ and $47 \mathrm{MHz}$, respectively. Moreover, the antenna maintains excellent read range and $\mathrm{CP}$ performance in different bending states. The developed tag antenna is, therefore, a promising solution for unnoticeable tracking operations. 


\section{REFERENCES}

[1] C. R. Medeiros, J. R. Costa, and C. A. Fernandes, "RFID Smart Shelf With Confined Detection Volume at UHF," IEEE Antennas and Wireless Propagation Letters, vol. 7, pp. 773-776, 2008.

[2] C. R. Medeiros and J. R. Costa and C. A. Fernandes, "RFID Reader Antennas for Tag Detection in Self-Confined Volumes at UHF," IEEE Antennas and Propagation Magazine, vol. 53, no. 2, pp. 39-50, April 2011.

[3] J. Lu and B. Chang, "Planar Compact Square-Ring Tag Antenna With Circular Polarization for UHF RFID Applications," IEEE Transactions on Antennas and Propagation, vol. 65, no. 2, pp. 432-441, Feb 2017.

[4] H. Chen, C. Tsai, C. Sim, and C. Kuo, "Circularly Polarized Loop Tag Antenna for Long Reading Range RFID Applications," IEEE Antennas and Wireless Propagation Letters, vol. 12, pp. 1460-1463, 2013.

[5] A. S. M. Sayem, R. B. V. B. Simorangkir, K. P. Esselle, R. M. Hashmi, and H. Liu, "A Method to Develop Flexible Robust Optically Transparent Unidirectional Antennas Utilizing Pure Water, PDMS and Transparent Conductive Mesh," IEEE Transactions on Antennas and Propagation, pp. 1-1, 2020.

[6] R. B. V. B. Simorangkir, D. Le, T. Bjrninen, A. S. M. Sayem, M. Zhadobov, and R. Sauleau, "Washing Durability of PDMSConductive Fabric Composite: Realizing Washable UHF RFID Tags," IEEE Antennas and Wireless Propagation Letters, vol. 18, no. 12, pp. 2572-2576, Dec 2019.

[7] H. H. Tran, S. X. Ta, and I. Park, "A Compact Circularly Polarized Crossed-Dipole Antenna for an RFID Tag," IEEE Antennas and Wireless Propagation Letters, vol. 14, pp. 674-677, 2015.

[8] H. Chen, S. Kuo, C. Sim, and C. Tsai, "Coupling-Feed Circularly Polarized RFID Tag Antenna Mountable on Metallic Surface," IEEE Transactions on Antennas and Propagation, vol. 60, no. 5, pp. 21662174, May 2012.

[9] H. Chen, C. Sim, and S. Kuo, "Compact Broadband Dual CouplingFeed Circularly Polarized RFID Microstrip Tag Antenna Mountable on Metallic Surface," IEEE Transactions on Antennas and Propagation, vol. 60, no. 12, pp. 5571-5577, Dec 2012.

[10] C. Cho, I. Park, and H. Choo, "Design of a Circularly Polarized Tag Antenna for Increased Reading Range," IEEE Transactions on Antennas and Propagation, vol. 57, no. 10, pp. 3418-3422, Oct 2009.

[11] F. Colombel, X. Castel, M. Himdi, G. Legeay, S. Vigneron, and E. M. Cruz, "Ultrathin metal layer, ITO film and ITO/Cu/ITO multilayer towards transparent antenna," IET Sci., Measure. Tech., vol. 3, no. 3, pp. 229-234, May 2009.

[12] S. Sheikh, M. Shokooh-Saremi, and M. Bagheri-Mohagheghi, "Transparent microstrip patch antenna based on fluorine-doped tin oxide deposited by spray pyrolysis technique," IET Microwaves, Antennas Propagation, vol. 9, no. 11, pp. 1221-1229, 2015.

[13] S. Y. Lee, M. Choo, S. Jung, and W. Hong, "Optically Transparent NanoPatterned Antennas: A Review and Future Directions," Applied Sciences, vol. 8, no. 6, 2018. [Online]. Available: https://www.mdpi.com/2076$3417 / 8 / 6 / 901$

[14] T. Jang, C. Zhang, H. Youn, J. Zhou, and L. J. Guo, "Semitransparent and flexible mechanically reconfigurable electrically small antennas based on tortuous metallic micromesh," IEEE Trans. Antennas Propag., vol. 65, no. 1, pp. 150-158, Jan 2017.

[15] A. Martin, X. Castel, O. Lafond, and M. Himdi, "Optically transparent frequency-agile antenna for X-band applications," Electronics Letters, vol. 51, no. 16, pp. 1231-1233, 2015.

[16] R. B. Green, M. Guzman, N. Izyumskaya, B. Ullah, S. Hia, J. Pitchford, R. Timsina, V. Avrutin, U. Ozgur, H. Morkoc, N. Dhar, and E. Topsakal, "Optically Transparent Antennas and Filters: A Smart City Concept to Alleviate Infrastructure and Network Capacity Challenges," IEEE Antennas and Propagation Magazine, vol. 61, no. 3, pp. 37-47, June 2019.

[17] W.-K. Kim, S. Lee, D. Hee Lee, I. Hee Park, J. Seong Bae, T. Woo Lee, J.-Y. Kim, J. Hun Park, Y. Chan Cho, C. Ryong Cho, and S.-Y. Jeong, "Cu Mesh for Flexible Transparent Conductive Electrodes," Scientific Reports, vol. 5, no. 1, p. 10715, 2015. [Online]. Available: https://doi.org/10.1038/srep10715

[18] A. S. M. Sayem, R. B. V. B. Simorangkir, K. P. Esselle, and R. M. Hashmi, "Development of Robust Transparent Conformal Antennas Based on Conductive Mesh-Polymer Composite for Unobtrusive Wearable Applications," IEEE Transactions on Antennas and Propagation, vol. 67, no. 12, pp. 7216-7224, Dec 2019.

[19] P. M. Bafrooei and L. Shafai, "Characteristics of single- and doublelayer microstrip square-ring antennas," IEEE Transactions on Antennas and Propagation, vol. 47, no. 10, pp. 1633-1639, Oct 1999.
[20] G. Marrocco, "The art of UHF RFID antenna design: impedancematching and size-reduction techniques," IEEE Antennas and Propagation Magazine, vol. 50, no. 1, pp. 66-79, Feb 2008.

[21] S. Sun, Z. Pan, F. K. Yang, Y. Huang, and B. Zhao, "A transparent silica colloidal crystal/PDMS composite and its application for crack suppression of metallic coatings," Journal of Colloid and Interface Science, vol. 461, pp. 136 - 143, 2016. [Online]. Available: http://www.sciencedirect.com/science/article/pii/S0021979715301855

[22] T. Bjrninen, J. Virkki, L. Sydnheimo, and L. Ukkonen, "Impact of recurrent stretching on the performance of electro-textile UHF RFID tags," in Proceedings of the 5th Electronics System-integration Technology Conference (ESTC), Sep. 2014, pp. 1-5.

[23] T. A. Milligan, Modern Antenna Design, 2nd ed. Wiley-IEEE Press, Jun. 2005, pp.18-24.

[24] J. Virkki, T. Bjrninen, S. Merilampi, L. Sydnheimo, and L. Ukkonen, "The effects of recurrent stretching on the performance of electro-textile and screen-printed ultra-high-frequency radio-frequency identification tags," Textile Research Journal, vol. 85, no. 3, pp. 294-301, 2015. [Online]. Available: https://doi.org/10.1177/0040517514545261 